\title{
Swirling granular solidlike clusters
}

\author{
Michael A. Scherer ${ }^{1, *}$ Karsten Kötter, ${ }^{2}$ Mario Markus, ${ }^{2}$ Eric Goles, ${ }^{3}$ and Ingo Rehberg ${ }^{1, \dagger}$ \\ ${ }^{1}$ Institut für Experimentelle Physik, Otto-von-Guericke-Universität, Postfach 4120, D-39016 Magdeburg, Germany \\ ${ }^{2}$ Max-Planck-Institut für molekulare Physiologie, Postfach 102664, D-44139 Dortmund, Germany \\ ${ }^{3}$ Departamento de Ingeniería Matemática, FCFM Universidad de Chile, Casilla 170-3, Santiago, Chile
}

(Received 23 April 1999; revised manuscript received 14 December 1999)

\begin{abstract}
Experiments and three-dimensional numerical simulations are presented to elucidate the dynamics of granular material in a cylindrical dish driven by a horizontal, periodic motion. The following phenomena are obtained both in the experiments and in the simulations: First, for large particle numbers $N$ the particles describe hypocycloidal trajectories. In this state the particles are embedded in a solidlike cluster ("pancake") which counter-rotates with respect to the external driving (reptation). Self-organization within the cluster occurs such that the probability distribution of the particles consists of concentric rings. Second, the system undergoes phase transitions. These can be identified by changes of the quantity $d E_{\mathrm{kin}} / d N$ ( $E_{\mathrm{kin}}$ is the mean kinetic energy) between zero (rotation), positive (reptation), and negative values (appearance of the totality of concentric rings).
\end{abstract}

PACS number(s): 81.05.Rm, 68.35.Rh, 83.10.Pp

\section{INTRODUCTION}

Although much theoretical progress has recently been achieved in uncovering the physics of granular media (see, e.g., Ref. [1]) a general theory for a consistent deduction of specific phenomena, e.g., finger patterns in granular flows [2], localized states in vertically vibrated layers [3], or stratification in a binary mixture of granular material [4], is still missing. To establish a theoretical description of granular matter is not merely an academic challenge; there is a demand to control granular materials in industrial processes involving basic materials, such as metallic powder, broken glass or sunflower grains, as well as final products, such as candy, champagne corks, or uranium tablets. To handle these materials different processes are involved: grinding [5], mixing [6], storing [7], transporting, and pouring [8]. During these processes there are a number of phenomena which reduce the production efficiency or increase the risk. Examples of those phenomena are arching, charging, density waves, dilatancy, fluidization, and segregation (see Ref. [9] for a review).

Lacking a general theory, in recent years numerical simulations have proved to be a powerful tool to model specific industrial applications or certain experimental setups [10]. To give a specific example, soft-sphere molecular dynamics simulations (MD) showed that material pulses at the outlet of vertical pipe conveyers are due to dynamical arches in the transportation belt [11]. On these conveyers the opaque rubber belt does not allow a visualization of the granular dynamics. This clearly demonstrates the advantages of simulating granular material.

Besides the already mentioned soft-sphere molecular dynamics simulation, other numerical techniques are used, e.g.,

\footnotetext{
*Present address: Quantum Institute, University of California, Santa Barbara, CA 93106.

${ }^{\dagger}$ Present address: Experimentalphysik V, Physikalisches Institut, Universität Bayreuth, 95440 Bayreuth, Germany.
}

event driven (ED) codes, Monte Carlo (MC) simulations, cellular automata, or a combination of ED and MC methods. Especially in MD and ED methods, the quality of the numeric code often relies on accurately modeling two things: first, the interaction of the particles with the confining boundaries of the setup; and second, the particle-particle interactions. To improve and test different models, simple experimental setups were recently considered to compare experimental and numerical results. A remarkable agreement between experiment and simulation is known, e.g., in the case of vertical oscillations of granular material [12] and for horizontal vibrations [13].

Difficulties in the numerical simulations normally arise due to the high computational effort in handling the large number of particles $\left(>10^{4}\right)$ in typical experimental systems. Therefore, we study an experimental setup which handles only a small number of particles $(<79)$; this avoids simplifications in the numerical model due to computational limitations. The system consists of a horizontally aligned cylindrical dish, which is driven externally in a horizontal swirling motion. It has been seen [14] that when there are only a few particles in the dish the spheres revolve about the center of the dish in the same direction as the external motion. This mode is called rotation. If the particles exceed a certain number they move counterclockwise (reptation). The observed phenomena depend sensitively on parameters like particle number, driving frequency $f_{d}$, and driving amplitude $A[14,15]$. Moreover, if $f_{d}>2.5 \mathrm{~Hz}$, material and surface properties of spheres play a role [16].

Experiments at high solid fractions give a visual impression that the ensemble of reptating particles behave like a pancake which is shaken in a frying pan to prevent it from burning. If, in fact, this picture of a rotating pancake is correct, then an individual particle should move on a hypocycloidal trajectory like a point on a solid disk does. In the present paper, we investigate the validity and limitations of this picture of a "granular pancake."

For simulating the system we use a more detailed mechanism than previously (in Ref. [14]), namely, a three- 


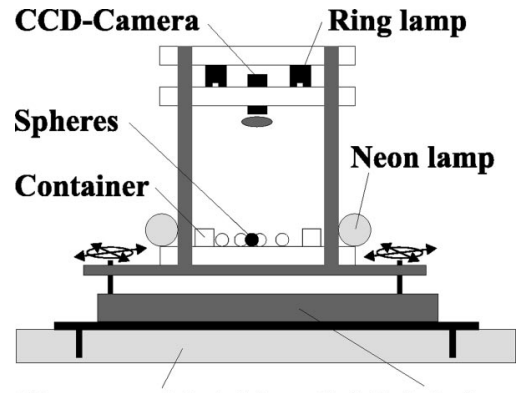

Heavy marble table Orbital shaker

FIG. 1. Experimental setup.

dimensional molecular dynamical model, where the rolling motion of the spheres and the interactions of the particles with the bottom and the wall of the dish are taken into account. To characterize the dynamics of the pancakelike state and of other modes, we determine the following from numerical and experimental data: (a) the short time trajectories of the spheres; (b) the probability densities of particle positions; (c) the mean frequencies of rotation around the center of mass and around the center of the dish; and (d) the mean kinetic energy of the particles.

\section{METHODS}

\section{A. Experiments}

The experimental setup shown in Fig. 1 is similar to the setup used in Refs. $[14,16]$. It consists of an adjustable reciprocating orbital shaker (Thermolyne AROS 160) which is mounted on a heavy marble table to avoid internal vibrations. During one oscillation period of the orbital shaker every point $(x, y)$ on the table moves on a circle in the laboratory frame according to $x=A \cos (\omega t)$ and $y=A \sin (\omega t)$. $A$ is the amplitude of the orbital motion, $\omega=2 \pi f_{d}$, and $f_{d}$ is the driving frequency of the shaker. $A$ is fixed to $9.53 \mathrm{~mm}$ and $f_{d}$ to $1.5 \mathrm{~Hz}$. A cylindrical dish is fastened on the swirling table by a holding device. The dish is milled out of Plexiglass. Its inner radius $R$ is $50 \mathrm{~mm}$. The height is $10 \mathrm{~mm}$. A white piece of paper between the top of the holding device and the bottom of the dish ensures a light background.

To characterize the bottom profile of the dish we use a surface analyzer (Talysurf Serie 120) with a resolution of $32.0 \mathrm{~nm}$. As seen in Fig. 2, the bottom profile has a slight monotonous drop towards the center of the dish. The height difference from the boundaries to the center, as determined in two different directions of the profile, is $125 \pm 5 \mu \mathrm{m}$.

As granular material we use $N$ spheres of two different types of soda lime glass, where $N$ ranges between 1 and 78 (the latter is the maximum number of spheres in the dish having contact with the bottom). A single particle consists of polished, black soda lime glass, and serves as a tracer sphere. The other spheres are uncolored and have a rough surface. Thus they diffuse the light and are not visible on the white background of the dish at bright illumination. Although the black sphere and the uncolored ones have different surface roughnesses it was shown in Ref. [16] for a different geometry (annular channel) that this does not influence the dynamics of the particles, namely, particle-wall and particle-particle interactions, if the particles are swirled at driving frequencies

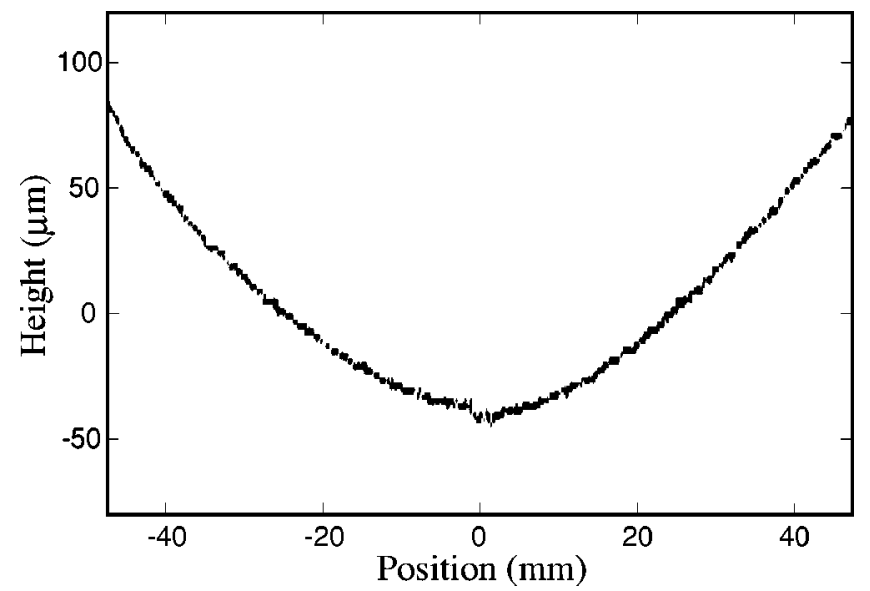

FIG. 2. Measured bottom profile of the cylindrical dish in the experiment.

below $2.5 \mathrm{~Hz}$. All particles have the same radius $r_{p}$ of 5 $\pm 0.01 \mathrm{~mm}$. The material density is $\rho=2.5 \mathrm{~g} \mathrm{~cm}^{-3}$. A CCD camera (Sony XC-77RR-CE) is mounted on top of the swirling table to take images in the comoving frame. The exposure time of the camera is set to $1 / 100 \mathrm{~s}$. The pixelsynchronous signals of the camera are grabbed for instant image processing by a real-time framegrabber board (BFPAT, Leutron Vision) which is controlled by a $90-\mathrm{MHz}$ personal computer. To obtain a homogeneous illumination we use two different light systems: First, there is an annular 22-W neon lamp (Philips TLE Cool White) which is fixed around the dish. Second, there is an additional ring lamp (Schott KL 1500 electronic) on top of the setup.

Figure 3 illustrates the procedure of detecting the center of the black tracer sphere. There are $N$ spheres in the circular

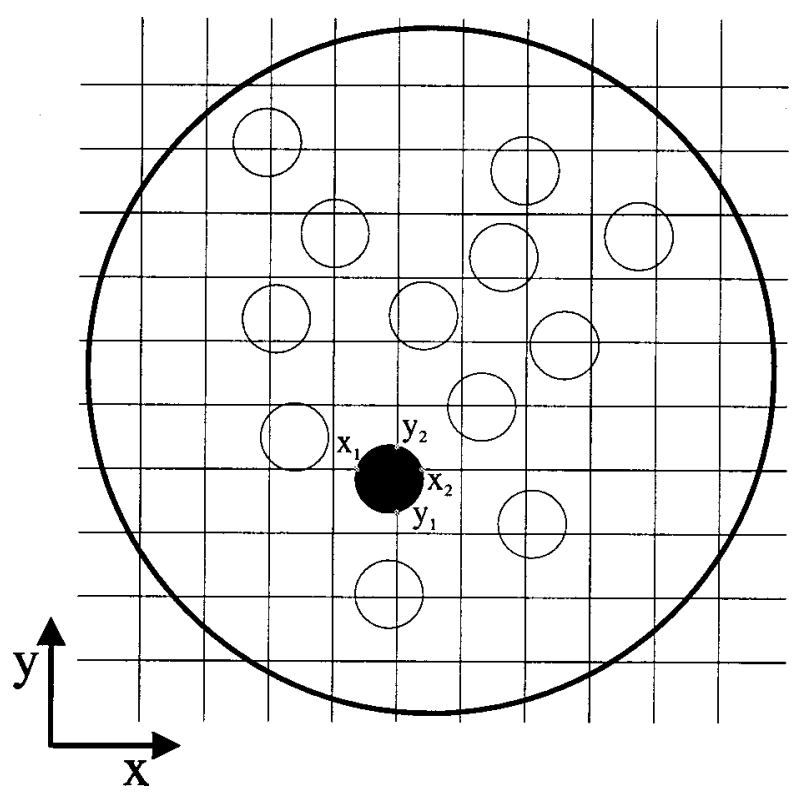

FIG. 3. Scheme demonstrating the procedure to detect the center of the single black tracer particle. The white spheres, whose boundaries are shown here, are not visible on the light background. The horizontal and vertical lines are used for the image processing algorithm. Only four points are necessary to calculate the coordinate of the center of the tracer particle: $x_{1}, x_{2}, y_{1}$, and $y_{2}$. The thick black circle shows the wall of the dish. 

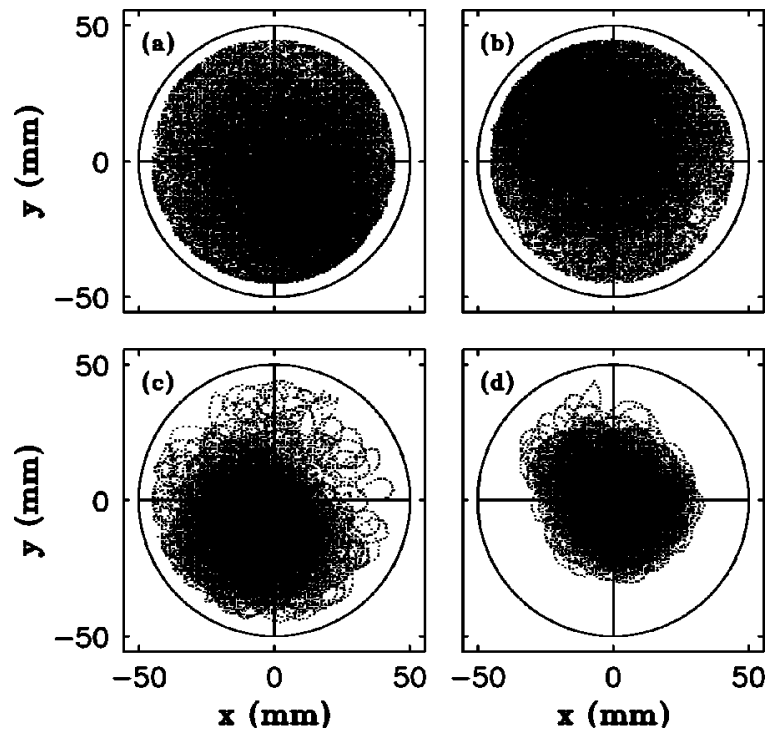

FIG. 4. Illustration of the alignment procedure of the dish. In (a), (b), and (c), the alignment is improved by putting small paper stripes below the dish, until the center of all detected particle coordinates is in the middle of the dish (d).

dish (thick circle). Although the $N-1$ light spheres are not visible in the image, their boundaries are shown in this figure (thin circles). The diameter of the dish corresponds to 214 pixels in the digitized image. Thus the diameter of a sphere is given by 21.4 pixels. Figure 3 shows a quadratic lattice. It consists of ten horizontal lines and ten vertical lines. The lattice spacing is set to 20 pixels. This guarantees that the black tracer sphere cuts twice, both a horizontal line and a vertical line of the lattice. An image with the size of $256 \times 256$ pixel is frozen every $20 \mathrm{~ms}$. To detect the center of the tracer sphere only the gray values along the horizontal and vertical lines have to be analyzed. This drastically reduces the computation time. We start to examine the gray values of the horizontal lines from left to right and the vertical lines from bottom to top. When the gray value is below a certain threshold $T$ the left boundary $x_{1}$ or the lower boundary $y_{1}$ of the tracer sphere is detected. When the value exceeds $T$ after having detected $x_{1}$ or $y_{1}$ we obtain the right boundary $x_{2}$ and the upper boundary $y_{2}$, respectively. Having detected these four coordinates the center of the tracer sphere $\vec{r}_{c}=\left(x_{c}, y_{c}\right)$ can be calculated by

$$
\vec{r}_{c}=\left(\begin{array}{l}
x_{c} \\
y_{c}
\end{array}\right)=\frac{1}{2}\left(\begin{array}{l}
x_{1}+x_{2} \\
y_{1}+y_{2}
\end{array}\right) .
$$

$x_{c}$ and $y_{c}$ are stored for later analysis. The procedure of detecting the center of the tracer sphere is repeated every 20 ms. Thus the trajectory of the sphere is tracked in real time. Because the data size of the detected point is small, this can be done over a long period of time to obtain good statistics.

It is seen that the trajectory of the tracer sphere for small $N$ depends sensitively on the horizontal alignment of the dish. Here the dip toward the dish's center (see Fig. 2) serves to level the setup. This is shown in Fig. 4. We use five spheres (which as shown below form a swarm) in the leveling process. The center of the spheres are detected over a period of $1310.70 \mathrm{~s}$ which corresponds to $2^{16}$ detections.
First the dish is aligned horizontally by using a circular spirit level. Figure 4(a) shows that the tracer sphere does not stay in the middle of the dish. It tends to collide with the wall of the dish (right bottom in the image). There it gains kinetic energy by hitting the moving wall. As time proceeds, the trajectory is blurred over the entire range of the dish, its mean value being displaced with respect to the center of the dish. By putting small pieces of paper below the dish, the time-averaged position of the tracer sphere is shifted to the dish's center [Figs. 4(b) and 4(c)]. The thickness of the paper is $0.11 \mathrm{~mm}$, which results in a change of the horizontal alignment of at least $2 \times 10^{-3} \mathrm{rad}$. This procedure gives reliable results as seen in Fig. 4(d), where the dish is now horizontally leveled. The tracer sphere stays around the center of the dish and does not hit the walls anymore.

In an experimental run we detected the trajectory of the tracer sphere for every number of spheres ranging from 1 to 78. Between each measurement we keep the driving constant, and add one particle at the wall of the cluster. We wait 2 min between each run to let the cluster dynamics relax. The detection process is started when the tracer sphere is again at the wall of the cluster.

\section{B. Numerical simulations}

The described system is simulated by using soft-sphere molecular dynamics. We made a three-dimensional model of $N$ spheres. $R, r_{p}$, and $\rho$ were set to the experimental values. A particle $i$ is described by its position $\vec{r}_{i}$, velocity $\vec{v}_{i}$, and angular velocity $\vec{\omega}_{i}$. Two particles $i$ and $j$ interact, if the distance between their centers of mass $\left|\vec{r}_{i}-\vec{r}_{j}\right|$ is smaller than the sphere diameter; the overlap is $\zeta=2 r-\left|\vec{r}_{i}-\vec{r}_{j}\right|$. The resulting normal force $\vec{F}_{n}^{(i)}$ on particle $i$ acts along the line connecting the centers of mass of the two interacting particles $i$ and $j: \vec{n}=\left(\vec{r}_{i}-\vec{r}_{j}\right) /\left|\vec{r}_{i}-\vec{r}_{j}\right|$. The force experienced by particle $j$ is deduced by exchanging subscripts $i$ and $j$. We choose a nonlinear repulsive force according to the Hertz theory with a viscoelastic dissipation of energy [17]:

$$
\vec{F}_{n}^{(i)}=\left[Y \zeta^{3 / 2}-\gamma_{n} \sqrt{\zeta}\left(\vec{v}_{i}-\vec{v}_{j}\right) \vec{n}\right] \vec{n}
$$

Since the impact velocity in our experiment is of the order of $0.1 \mathrm{~m} / \mathrm{s}$ and thus smaller than the yield velocity, which is of order $1 \mathrm{~m} / \mathrm{s}$ [18], we assume that there are no plastic deformations in our material, and only the viscoelastic dissipation of energy is the relevant mechanism in a binary collision. Equation (2) implies that the coefficient of restitution decreases when the collision velocity increases and is in good agreement with experimental data of two particle collisions (For a comparison of numerical simulations and experimental data, see Refs. [19,22]). The stiffness of the particles is chosen to be $Y=1.0 \times 10^{5} \mathrm{~kg} \mathrm{~m}^{-1 / 2} \mathrm{~s}^{-2}$ and the energy is dissipated through $\gamma_{n}=30.0 \mathrm{~kg} \mathrm{~m}^{-1 / 2} \mathrm{~s}^{-1}$, leading to a restitution coefficient of the order of 0.67 for a velocity of 0.1 $\mathrm{m} \mathrm{s}^{-1}$. This value is smaller than the value 0.97 , measured by Foerster et al. [20] at $1.0 \mathrm{~m} \mathrm{~s}^{-1}$, but leads to a robust agreement between experimental and simulational quantities. Furthermore a shear force $\vec{F}_{s}^{(i)}$ acting perpendicular to the normal force is added: 

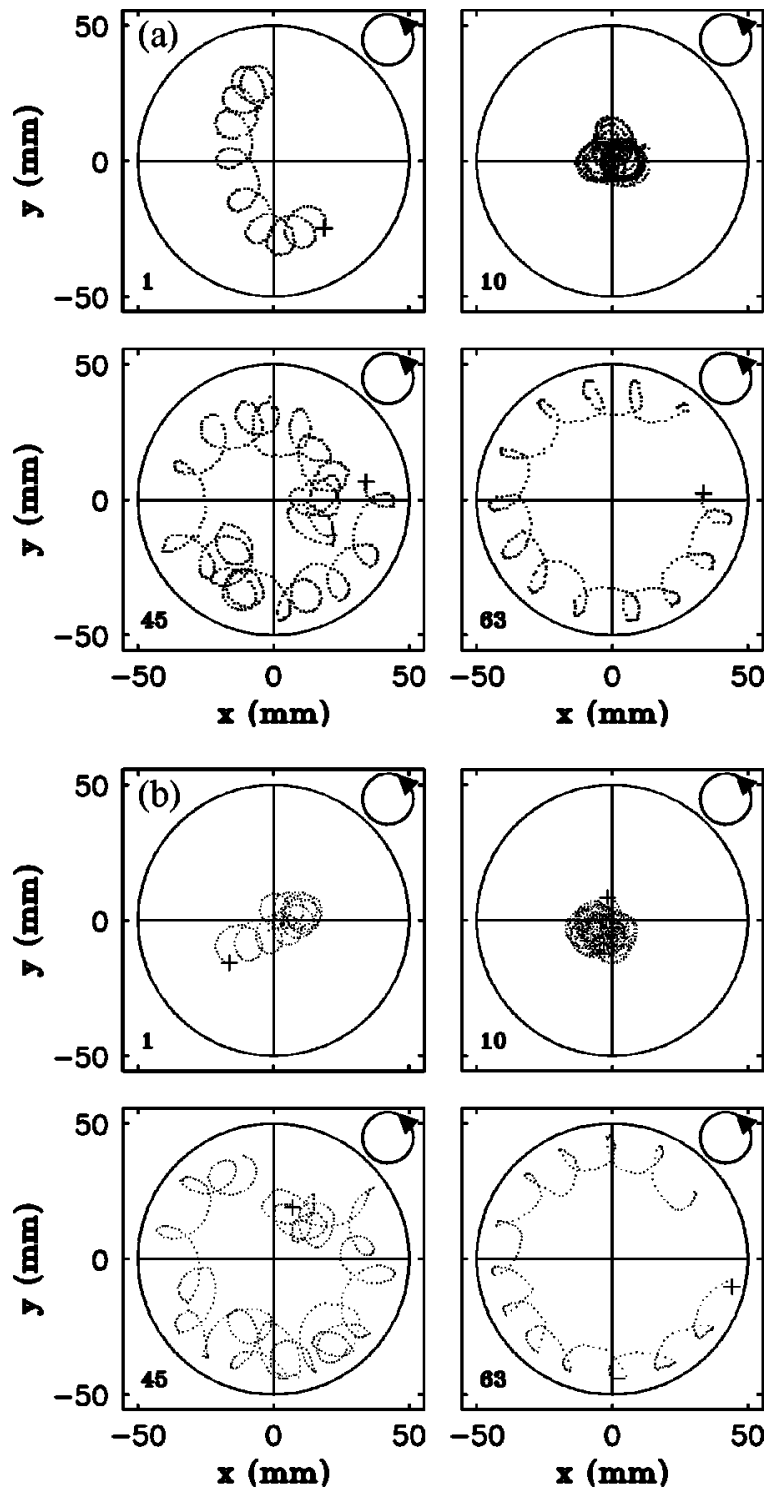

FIG. 5. Experimental (a) and numerical (b) trajectories of the center of a particle. The center of the tracer particle is plotted every $20 \mathrm{~ms}$. The cross marks the starting point of the trajectory. The number in the lower left corner of each image denotes the total number $N$ of spheres in the dish. A circle with an arrow in the upper right corner gives the sense of excitation caused by the orbital motion of the dish. The radius of this circle is the amplitude of the orbital motion. Due to different velocities of the tracer sphere at different solid fractions we display different time periods $t$ to avoid overlapping of the trajectories: $N=1, t=6 \mathrm{~s} ; N=10, t=20 \mathrm{~s} ; N$ $=45, t=14 \mathrm{~s}$; and $N=63, t=8 \mathrm{~s}$.

$$
\vec{F}_{s}^{(i)}=-\frac{\vec{v}_{s}}{\left|\vec{v}_{s}\right|} \min \left\{\mu_{s}\left|\vec{F}_{n}^{(i)}\right|, \gamma_{s}\left|\vec{v}_{s}\right|\right\}
$$

The first term describes the Coulomb sliding friction and the second term the viscous friction, respectively [21]. In general, this also leads to a realistic reproduction of experimental data as shown in Refs. [19,22]. The parameters of the shear force are set to $\gamma_{s}=20.0 \mathrm{~kg} \mathrm{~s} \mathrm{~s}^{1}$ and $\mu_{s}=0.45$. These values were chosen to reproduce first the experimental data in Figs. 5 and 8, and second the experimental data reported in Ref. [14]. It is observed that a lower value of $\mu_{s}$ destroys the loops of the trajectories by causing a more sliding than rolling movement of the particles. A higher value leads to stronger interactions of neighboring particles and therefore to a different frequency of rotation. Furthermore, a different value of $\gamma_{s}$ shifts the rotation frequency slightly. The shear velocity $\vec{v}_{s}$ is given in the following way [23]: $\vec{v}_{s}=\vec{v}_{i}-\vec{v}_{j}$ $-\left[\left(\vec{v}_{i}-\vec{v}_{j}\right) \vec{n}\right] \vec{n}+r_{p} \vec{n} \times\left(\vec{\omega}_{i}+\vec{\omega}_{j}\right)$.

The interaction of a particle with the boundaries (bottom and wall) of the dish are simulated by a real particle and a virtual particle, where the latter plays the role of the boundary. The virtual particle has the same velocity as the simulated boundary, and the same properties as the real particle. It is positioned in a way that its normal vector $\vec{n}$ is perpendicular to the corresponding boundary. Due to the constant distance between the particle and the bottom of the dish, there is no energy dissipation in this interaction. However, dissipation occurs by introducing an additional force, namely, rolling friction,

$$
\vec{F}_{r}=\mu_{r} \frac{\left|\vec{F}_{n}^{(i)}\right|}{r_{p}} \cdot \frac{\vec{v}-\vec{v}_{i}}{\left|\vec{v}-\vec{v}_{i}\right|}
$$

which is only acting on particle $i$ if there is an interaction between the particle and the bottom of the dish. Rolling friction only plays a relevant role in dissipating energy at very low particle numbers $(N<4)$. It prevents the particles of moving with unrealistic high velocities caused by wall collisions and missing damping due to rare particle-particle collisions as observed in the experiment. $\vec{F}_{r}$ is added to the other forces [Eqs. (2) and (3)] if there is an interaction between a particle and the bottom of the dish. $\mu_{r}$ was optimized by comparing experiments and simulations in Figs. 5-8; best agreement was obtained for $\mu_{r}=3.0 \times 10^{-5} \mathrm{~m} . \vec{v}$ is determined by the swirling motion of the table, and gives the velocity of every point on the dish: $\vec{v}=(-A \omega \sin (\omega t), A \omega \cos (\omega t), 0)$. Furthermore, the height profile of the dish (see Fig. 2) is adapted by a parabolic curvature of the simulated bottom $h(r)=a r^{2}$, where $r$ is the distance from the center of the dish, and $a$ is set to $0.04 \mathrm{~m}^{-1}$. The roughness of the dish is simulated by adding a stochastically determined quantity $q$ to the center of the virtual sphere, and thus to the bottom height at each time step. Best agreement with the experiments was obtained by choosing an equally distributed $q$ with $-10 \mu \mathrm{m}<q<10 \mu \mathrm{m}$, i.e., by assuming a somewhat larger value than in Fig. 2. Finally the gravity $g=9.8 \mathrm{~m} \mathrm{~s}^{-2}$ is acting on the particles along the negative $z$ axis. The resulting differential equations for the translation and the rotation are solved numerically by a Gearpredictor-corrector algorithm [24] of sixth order with an integration time step of $5.0 \times 10^{-5} \mathrm{~s}$.

In a simulational run the system is allowed to relax for 10 $\mathrm{s}$. Then the positions and velocities of all simulated particles are stored for later analysis every $20 \mathrm{~ms}$ (as in the experiment), over a total time of $100 \mathrm{~s}$.

\section{RESULTS AND DISCUSSION}

\section{A. Particle trajectories}

For a first and qualitative description of the dynamics of the cluster, we look at short time series of the trajectory of 

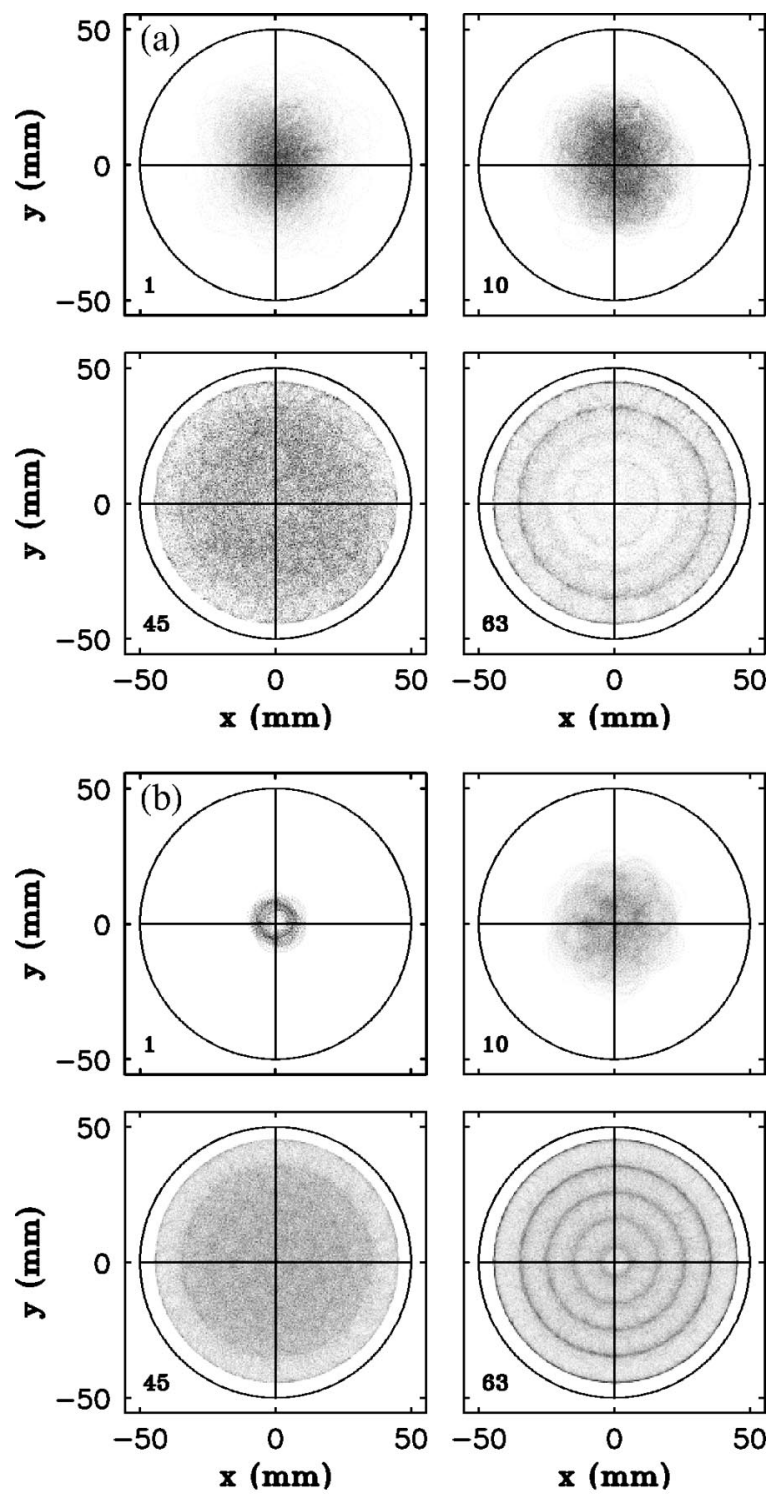

FIG. 6. Probability distribution of the particle position (a) in the experiments and (b) in the numerical simulations. The intensity in each image is scaled from 0 to 255 . Thus dark regions refer to high probability distributions, and light spots to low ones. The number of particles $N$ is given in the lower left corner of each image.

the tracer sphere. In Fig. 5(a) we show examples of the experimental trajectories for different particle numbers. These compare well with trajectories of the corresponding numerical simulation [Fig. 5(b)].

For $N=1$ the path of the tracer sphere consists of a curved motion superimposed with loops. The loops reflect the driving frequency of the swirling table. The curvature of the path is due to the bottom profile of the circular dish.

For small $N$ the particles move around in a swarm, i.e., the particles stay close together and perform the same circular motion. Thus neighboring spheres have the same sense of spinning motion as they roll on the dish. It follows that two touching spheres have opposite velocities at their contact point. Therefore, friction between touching spheres is high and they slow down, stay close together, and build the observed swarm. If a sphere collides with the wall of the circular dish, its velocity significantly increases. The sphere

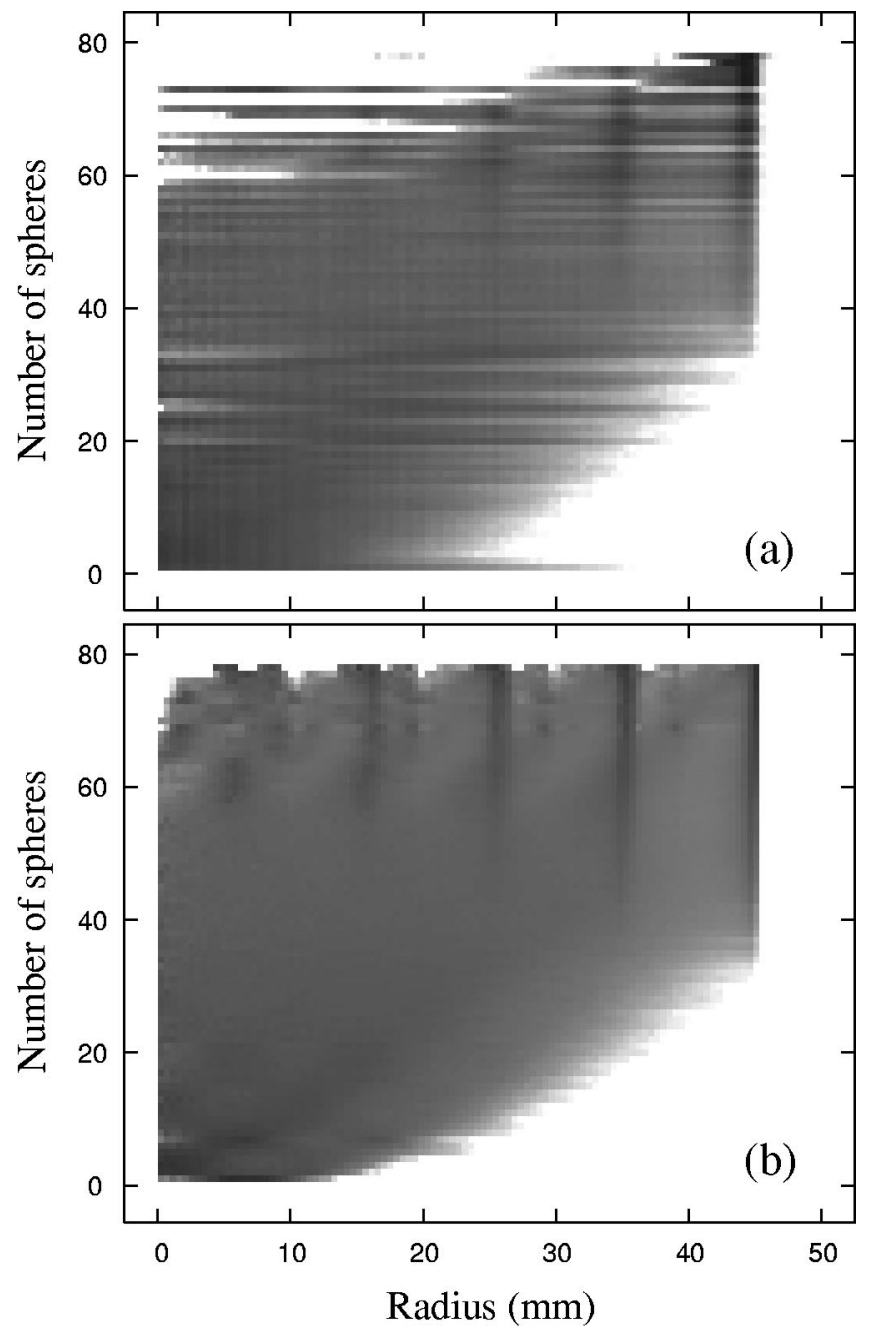

FIG. 7. Dependence of the probability distribution on the radial position of the dish and the number of particles in the experiment (a) and in the numerical simulation (b). The probabilities correspond to the azimuthal average. The logarithm of the probabilities is mapped to a grey scale; white corresponds to a low probability, and dark to a high probability. All probabilities below $10^{-4}$ are set to 0 .

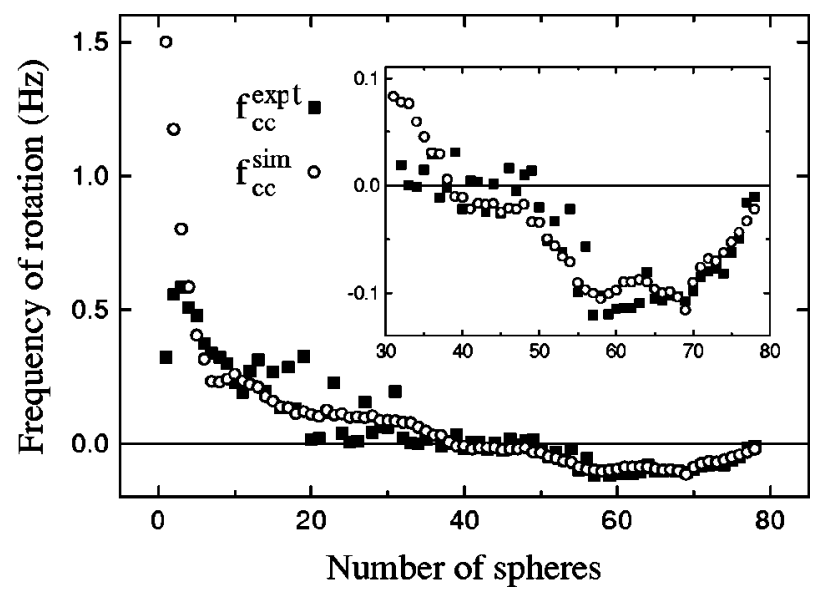

FIG. 8. Comparison between experimentally and numerically determined dependence of the mean frequency of rotation of the cluster (around the center of the dish) on the number of spheres. 
then hits the cluster and reduces its speed significantly. By sound emission it is noticeable that the additional kinetic energy is dissipated by multiple collisions inside the cluster. Because of the dip in the bottom profile and the abovementioned horizontal alignment of the dish the cluster stays in the neighborhood of the dish's center. This is seen in Figs. 5 (a) and 5(b) for $N=10$.

For $N=30$ it is observed that the number of collisions with the wall increases, as the size of the cluster becomes larger. For $N>35$ the particles starts to reptate as described in Ref. [14]. Reptation means that the particles rotate clockwise although the driving of the system is a counterclockwise motion. Examples of reptation are seen in Figs. 5(a) and 5(b) for 45 spheres. In this case the mixing of particles is high, i.e., the tracer particle does not stay in the outer region where it started.

For 63 particles [Figs. 5(a) and 5(b)] the path of the tracer sphere resembles a hypocycloid. Hypocycloids occur when a solid disk unrolls at the inner wall of a circle. Their shapes depend on the ratio between the radius of the disk and the radius of the circle. Furthermore it is important which point on the disk is tracked to obtain the trajectory: loops are observed for a point inside the disk, whereas cusps appear for a point on the wall of the disk. The outer flattening of the loops for $N=63$ in Figs. 5(a) and 5(b) is due to slipping at the wall. The loops become cusps when $N$ is increased in our experimental and numerical setup. At high $N$ it is observed that the tracer particle is confined to the outer regions of the cluster, although it sometimes jumps to inner regions. Finally for $N$ $=78$, where we have the highest possible solid fraction, the dynamics is almost frozen and the trajectory just consists of a thin annulus.

\section{B. Probability distributions}

We look at the long time behavior of the particles, and we calculate the probability distribution $P=P(\vec{r}, N)$ for each image pixel $\vec{r}=(x, y)$ in our dish. In the experiments only the position of the tracer particle is analyzed, whereas in the simulation the positions of all particles are considered. Figures 6(a) and 6(b) show probability distributions for the experiments and the numerical simulations, respectively.

The probability distribution for $N=1$ is related to the bottom profile of the dish. In the simulations we assumed a parabolic bottom profile which is more perfect than in the experiments. It follows (in spite of the large value of the stochastic parameter $q$ ) that the sphere circles around the center of the dish, which implies an annular particle distribution with a minimum at $r=0$. For few particles (e. g., $N$ $=10$ ) we observe that the probability distribution becomes more homogeneously distributed around the center. This is due to the above-mentioned swarmlike behavior of the spheres, and because multiple collisions dissipate the kinetic energy.

For an increasing number of particles, collisions with the wall of the dish become more abundant. In Fig. 6 we show an example for $N=45$. The probability distribution is high at $r \approx 45 \mathrm{~mm}$ because there are collisions with the wall, and the spheres change their direction. Closer to the center there is an annular zone where $P$ is smaller, because of the higher velocities of the particles after collisions with the wall. In the central region $P$ is larger because, due to collisions between spheres, they slow down and cluster. At higher solid fractions we observe a ring structure in the probability distribution (target pattern). This is seen for $N=63$ in Fig. 6 .

While Fig. 6 shows the behavior for some particle numbers only, Fig. 7 summarizes the dynamics for all particle numbers. The gray scale values in Fig. 7 are determined by the azimuthal average of $P(\vec{r}, N)$ :

$$
G(r, N)=\int_{0}^{2 \pi} P(r, \varphi, N) d \varphi .
$$

We map $\ln G(r, N)$ to a gray scale with 256 levels.

Figures 7(a) and 7(b) show the experimental results and the numerical simulations, respectively. In the experimental situation we see a sharp decrease of the size of the occupied region as $N$ increases from 1 to 3 . This is due to the abovementioned energy dissipation by multiple collisions and clustering. At higher $N$ the occupied area increases approximately linearly with $N$. As $N$ is increased further, first the outer rings start to emerge and then the inner rings. Finally a target pattern is formed as given in Fig. 6. Closer inspection shows that the regions in which rings have emerged can be considered as solidlike bodies rotating in a nearly concerted way. In each ring, the particles describe a hypocycloidal motion. Such a motion consists of alternating large inner speeds (implying small $G$ ) and smaller outer speeds (implying larger $G$ ). This leads to the observed annuli of $P$. For $N$ $>57$, all rings are formed. The whole (reptating) cluster can then be approximated by a solid disk and this state is what we compare with a "pancake."

A comparison of Figs. 7(a) and 7(b) shows a qualitative agreement between the experimental and numerical results. The disagreement in both images at low radii and high particle numbers is due to the fact that the time series in the experimental runs are too short for high particle numbers. In fact, since the tracer sphere always starts at the wall of the cluster, it does not have enough time to travel toward the central region at high $N$.

\section{Global quantities}

\section{Different mean frequencies of rotation}

In Sec. III B we carried out a qualitative comparison of experiments and simulations. We now want to compare the results in a quantitative way by determining global characterizing quantities. For this purpose, we first extract the rotation frequency of the cluster from the time series in the experiments and in the simulations. Three different frequencies may be considered: the rotation frequency of the cluster around the center of the dish $\left(f_{\mathrm{cc}}\right)$, the rotation frequency of the cluster around the center of mass of the cluster $\left(f_{\mathrm{cm}}\right)$, and finally the rotation frequency of the center of mass of the cluster around the center of the dish. The latter was analyzed in the simulations, and was seen to be equal to the driving frequency of the table for every particle number, while the first two have a more complicated dependence on $N$, which will be discussed here in more detail.

Figure 8 compares the rotation frequency of the cluster around the center of the dish in the numerical simulation $f_{\mathrm{cc}}^{\mathrm{sim}}$ with that in the experiment $f_{\mathrm{cc}}^{\text {expt }}$. In the simulations the mean 
frequency of rotation is calculated by

$$
f_{\mathrm{cc}}^{\mathrm{sim}}=\frac{1}{2 \pi N}\left\langle\sum_{i=1}^{N} \frac{\left|\vec{r}_{i} \times \vec{v}_{i}\right|}{\left|\vec{r}_{i}\right|^{2}}\right\rangle_{t},
$$

where $\vec{r}_{i}$ and $\vec{v}_{i}$ refer to the reference frame of the moving dish.

In the experiments we analyze the time series of the tracer sphere to derive $f_{\mathrm{cc}}^{\text {exp }}$. Here we suppose that the time scale of each measurement is long enough to represent the rotation frequency of the cluster. When the solid fraction $\left(N r_{p}^{2} / R^{2}\right)$ is low the particles mix, and thus the tracer sphere is present in all regions of the cluster. At high solid fractions mixing is not important, and it is assumed that the angular behavior of the spheres is the same regardless of the position of the sphere in the cluster.

Figure 8 shows the rotation mode $\left(f_{\mathrm{cc}}>0\right)$ for low particle numbers, and the transition to the reptation mode $\left(f_{\mathrm{cc}}\right.$ $<0$ ) for higher particle numbers. By comparing $f_{\mathrm{cc}}^{\mathrm{sim}}$ and $f_{\text {cc }}^{\text {expt }}$ we find a good agreement for $N>35$ where the cluster is in the reptation mode. For smaller values the scatter of the experimental data is larger. In addition, there is a noticeable disagreement for $N<4$. The higher value from simulations is due to the fact that a single particle circles around the center of the dish because of the parabolic profile of the bottom [see $N=1$ in Fig. 6(b)]; the higher randomness of the trajectories in the experiments decreases the rotation frequency.

The mean frequency of rotation of all simulated particles around the center of mass of the cluster is given by

$$
f_{\mathrm{cm}}^{\mathrm{sim}}=\frac{1}{2 \pi N}\left\langle\sum_{i=1}^{N} \frac{\vec{r}_{i}^{*} \times \vec{v}_{i}^{*}}{\left|\vec{r}_{i}^{*}\right|^{2}}\right\rangle_{t} .
$$

$\vec{r}_{i}^{*}$ and $\vec{v}_{i}^{*}$ are the position and velocity of particle $i$ relative to the cluster:

$$
\vec{r}_{i}^{*}=\vec{r}_{i}-\frac{1}{N} \sum_{j=1}^{N} \vec{r}_{j}, \quad \vec{v}_{i}^{*}=\vec{v}_{i}-\frac{1}{N} \sum_{j=1}^{N} \vec{v}_{j}
$$

In Fig. 9 we compare $f_{\mathrm{cm}}^{\mathrm{sim}}$ with $f_{\mathrm{cc}}^{\mathrm{sim}}$. Here it is seen that in the rotation mode $f_{\mathrm{cm}}^{\mathrm{sim}}$ is much smaller than $f_{\mathrm{cc}}^{\mathrm{sim}}$. This means that it is the rotation of the particles around the center of the dish rather than the overall spin of the cluster which contributes to the appearance of what is called the rotation mode. However, at high solid fractions there is a convergence of both measures caused by the fact that the cluster radius approaches the dish radius.

$f_{\mathrm{cc}}^{\mathrm{sim}}$ and $f_{\mathrm{cm}}^{\mathrm{sim}}$ are compared with an analytical value which we call the frequency of rotation of the hypocycloid $f_{\text {hyp }}$. As already mentioned, the global motion of the cluster at high solid fractions appears as if a solid disk unrolls at the inner wall of a circle. Neglecting the slip between the disk and the wall, any point in the disk thus has a frequency of rotation around its center of mass given by

$$
f_{\text {hyp }}=\left(1-\frac{R}{r_{d}}\right) f_{d} \text {. }
$$

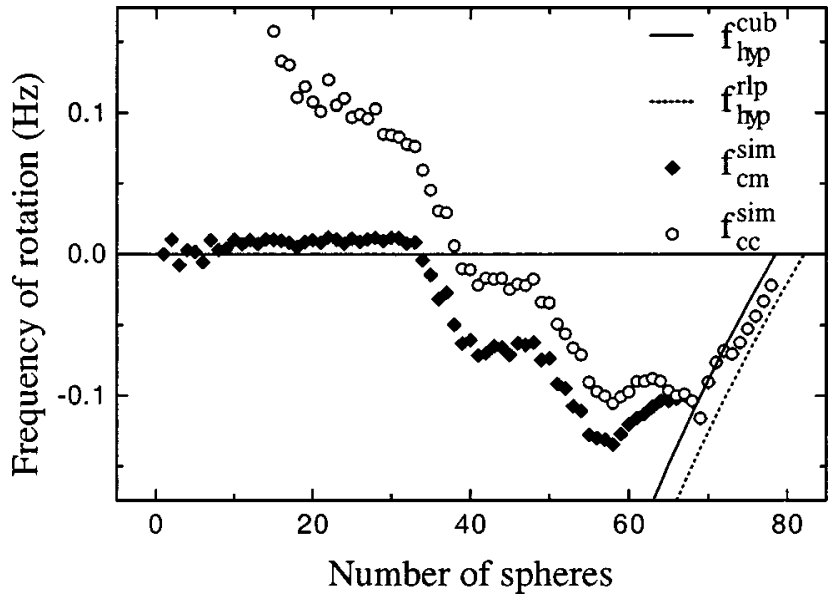

FIG. 9. Comparison between two different numerically determined mean frequencies of rotation. The open circles show the frequency of rotation $f_{\mathrm{cc}}^{\mathrm{sim}}$ as given in Fig. 8 and the closed squares describe the rotation of the particles around the center of mass $(\mathrm{cm})$ of the cluster. For $N>65$, the closed squares are almost not visible because they coincide with the open circles. The solid line describes the frequency of rotation $f_{\text {hyp }}^{\text {cub }}$ obtained by the hypocycloid model [Eq. (10)] with a cubic packing of the spheres, whereas $f_{\text {hyp }}^{\text {rlp }}$ is due to a random loose packing.

If we assume that the particles cluster as a disk, its radius $r_{d}$ is given by $\sqrt{N / p} r_{p}$. Hence we obtain

$$
f_{\text {hyp }}=\left(1-\sqrt{\frac{p}{N}} \frac{R}{r_{p}}\right) f_{d} .
$$

We distinguish between two different packing densities $p$ : First we assume a cubic packing (cub) of the cluster with $p_{\text {cub }}=\pi / 4$, and second a random loose packing structure (rlp) with $p_{\text {rlp }}=\pi^{2} / 12[25]$.

Figure 9 shows both $f_{\text {hyp }}^{\mathrm{cub}}(N)$ and $f_{\text {hyp }}^{\text {rlp }}(N)$. It is seen that the data points at high solid fractions $(N>68)$ are close to cubic and random loose packing structures. This confirms the idea of a solid "granular pancake" at high $N$. Although the motion of a solidlike pancake is applicable for $N>57$, i.e., if all rings are formed, the geometric approximation (10) is only valid if $p$ is nearly independent of $N$, which is the case for $N>68$.

\section{Mean kinetic energy}

In addition to the different frequencies of rotation of the cluster, we now use a thermodynamical quantity to characterize the behavior of the cluster in dependence on $N$, namely, the mean kinetic energy $E_{\text {kin }}$. In the numerical simulation $E_{\text {kin }}$ is given by

$$
E_{\mathrm{kin}}^{\mathrm{sim}}(N) \equiv\left\langle\frac{m}{2 N} \sum_{i=1}^{N}\left(\vec{v}_{i}(t)\right)^{2}\right\rangle_{t},
$$

where $\vec{v}_{i}(t)$ refers to the reference frame of the moving dish.

In the experiment only the center $\vec{r}_{c}$ of the tracer sphere is known at given time intervals $\Delta t=20 \mathrm{~ms}$. In this case the mean kinetic energy is given by 


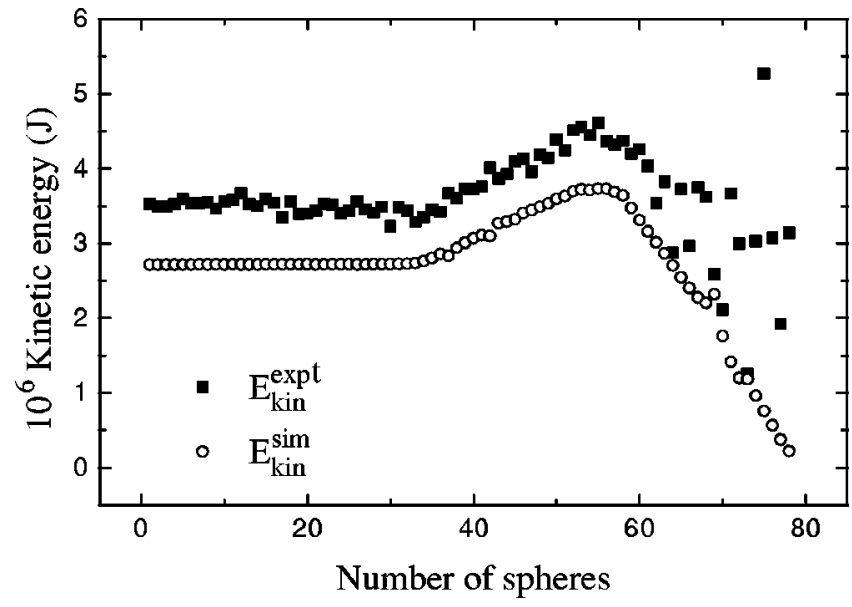

FIG. 10. Dependence of the mean kinetic energy $E_{\text {kin }}$ on the number of spheres. $E_{\text {kin }}^{\text {expt }}$ is obtained from the experiments and $E_{\mathrm{kin}}^{\mathrm{sim}}$ by the molecular dynamics simulations.

$$
E_{\mathrm{kin}}^{\operatorname{expt}}(N) \equiv\left\langle\frac{m}{2 N}\left(v_{c}(t)\right)^{2}\right\rangle_{t}
$$

The velocity of the tracer sphere $v_{c}$ is calculated in the following way: $v_{c}(t)=\left|\vec{r}_{c}(t+\Delta t)-\vec{r}_{c}(t)\right| / \Delta t$.

Figure 10 shows that the kinetic energy from simulations compares well with that from experiments. There are, however, two discrepancies: the experimental values are higher than the numerical ones, and the scatter in the experimental data is higher for large $N$. The first discrepancy is explained by the fact that the tracer sphere always starts in the outer regions of the cluster where the kinetic energy is higher, and stays predominantly far from the center. It is left as an open question why this discrepancy does not vanish at small $N$, where particle mixing is more probable. The large experimental scatter is explained by the fact that in some cases the particles remain confined in certain regions of the cluster, while in other cases perturbations drive particles into other regions. These different results obtained from confined and nonconfined particles are averaged out in the simulations, in which the whole particle ensemble is considered. In spite of these discrepancies, Fig. 10 allows one to discern three dynamic behaviors, which are more clearly defined than in Fig. 9 and may be identified here as granular phases. The first one $\left(d E_{\mathrm{kin}} / d N=0\right)$ corresponds to the isolation mode with missing particle-wall collisions and constant $f_{\mathrm{cm}}^{\mathrm{sim}}$. The transition to the second one $\left(d E_{\mathrm{kin}} / d N>0\right)$ corresponds to the transition to reptation, where the mean kinetic energy increases with $N$ due to an increasing energy input from the wall. The third one $\left(d E_{\mathrm{kin}} / d N \leqslant 0\right)$ reflects the increasing dissipation due to particle-particle collisions; it manifests itself in the appearance of the totality of concentric rings.

\section{CONCLUSIONS}

We used experimental and numerical setups to explore a cluster of spheres in a cylindrical dish, which is driven in an orbital motion. In the experiment we followed the trajectory of a single particle. This was done by image processing and using a simple algorithm to extract and store the position of the tracer bead for a long time. In the numerical setup we chose a three-dimensional molecular dynamics simulation. Here the rotation of the particles, the interactions with the wall, and the bottom profile were taken into account.

By analyzing the trajectories we obtained information on the short- and long-time behaviors of the particles. Moreover we determined global characterizing quantities of the cluster, namely, two different mean frequencies of rotation and the kinetic energy. The short-time trajectories of single spheres in the experiments and in the numerical simulations showed that the system is very sensitive to the bottom profile of the cylindrical dish and the horizontal alignment.

A central question of the investigations was whether the cluster dynamics can be captured by a solid-body ("pancake") model. The experimental and numerical findings corroborate this view for large $N$. First, the trajectories of the particles in the experiments and in the simulations showed features known from hypocycloids. Second, we found a good agreement of $f_{\mathrm{cc}}$ and $f_{\mathrm{cm}}$ with a theoretically derived dependency of the frequency of rotation on $N$ which is based on the hypocycloid model. Despite this there was no evidence of a hypocycloidal behavior for small $N$. Here the particles in the cluster move around the center of the dish in a disordered swarm. This clustering is due to the dissipation of kinetic energy by multiple collisions.

By analyzing the long-time series of the tracer sphere in the experiments and all particle positions in the simulations, we observed a target pattern in the probability distributions of the spheres for high solid fractions. This distribution is due to self-organization into rings, all of them describing hypocycloidal trajectories.

For the global behavior of the cluster we demonstrated that it is important to distinguish between two different frequencies of rotation: one around the center of the circular dish and one around the center of mass of the cluster. For the first case there is a good quantitative agreement between the experimental and numerical findings. By comparing $f_{\mathrm{cc}}$ and $f_{\mathrm{cm}}$ in the numerical simulations we showed that it is mainly the rotation of the cluster around the center of the dish which contributes to the rotation mode; in contrast, the reptation mode is characterized by a convergence of both frequencies for increasing $N$.

Finally, we calculated the mean kinetic energy $E_{\text {kin }}$ and found phase transitions for $N$ values agreeing between experiments and simulations. In fact, $d E_{\mathrm{kin}} / d N$ clearly characterizes the transitions between rotation, reptation, and full ring formation.

By comparing experimental and numerical results in a simple system we established a reliable numerical code for simulating granular dynamics in three dimensions, to which we attach importance for the following two reasons: First, the numerical simulations can guide the experimental work to uncover the underlying physics for the observed transitions. Second, in a more general view, the extractable thermodynamic quantities - in our work the mean kinetic energy - may support the establishment of a basic theory of granular media, and in particular of granular phase transitions.

\section{ACKNOWLEDGMENTS}

This work has been supported by the Deutsche Forschungsgemeinschaft (Grant Nos. Re 588/11-1,2 and No. Ma 
629/4) and FONDAP program in Particle Systems (Chile). We gratefully acknowledge B. Eckhardt and W. van Saarloos for bringing our attention to the hypocycloid model. Moreover, it is a pleasure to thank G.
Straßburger, K. Kassner, C. Krülle, and K. Kuehn for valuable contributions. We also owe much to T. Bock, H. Brunn, and T.-J. John for performing surface roughness measurements.
[1] H. Hayakawa, S. Yue, and D. C. Hong, Phys. Rev. Lett. 75, 2328 (1995); S. J. Linz, Phys. Rev. E 54, 2925 (1996); B. C. $\mathrm{Eu}$ and H. Fahrat, ibid. 55, 4187 (1997).

[2] O. Pouliquen, J. Delour, and S. B. Savage, Nature (London) 386, 816 (1997).

[3] P. Umbanhowar, F. Melo, and H. L. Swinney, Nature (London) 382, 3838 (1996).

[4] H. A. Makse, S. Havlin, P. R. King, and E. Stanley, Nature (London) 386, 379 (1997).

[5] L. Rolf, Zement-Kalk-Gips 3, 117 (1993); W. Scheibe, ibid. 3, 123 (1993).

[6] H. Rumpf, Particle Technology (Chapman and Hall, London, 1990).

[7] P. Martens, Silo-Handbuch (Ernst \& Sohn, Berlin, 1988).

[8] J. Tomas, CHEMTECH 43, 307 (1991).

[9] H. M. Jaeger, S. R. Nagel, and R. P. Behringer, Rev. Mod. Phys. 68, 1259 (1996).

[10] H. J. Herrmann, in Mobile Particulate Systems, edited by E. Guazelli and L. Oger (Kluwer, Dordrecht, 1997), pp. 281-304.

[11] T. Gröger, in Friction, Arching, Contact Dynamics, edited by D. E. Wolf and P. Grassberger (World Scientific, Singapore, 1997), pp. 225-289.

[12] S. Luding, E. Clément, A. Blumen, J. Rajchenbach, and J. Duran, Phys. Rev. E 50, R1762 (1994); C. Bizon, M. D. Shattuck, J. B. Swift, W. D. McCormick, and H. L. Swinney, Phys. Rev. Lett. 80, 57 (1998).

[13] G. H. Ristow, G. Strassburger, and I. Rehberg, Phys. Rev. Lett. 79, 833 (1997)
[14] M. A. Scherer, V. Buchholtz, T. Pöschel, and I. Rehberg, Phys. Rev. E 54, R4560 (1996).

[15] A. Schinner, M. Scherer, I. Rehberg, and K. Kassner, in Physics of Dry Granular Media, edited by H. J. Herrmann, J.-P. Hovi, and S. Luding (Kluwer, Dordrecht, 1998), pp. 639-644.

[16] M. A. Scherer, T. Mahr, A. Engel, and I. Rehberg, Phys. Rev. E 58, 6061 (1998).

[17] G. Kuwabara and K. Kono, Jpn. J. Appl. Phys. 26, 1230 (1987).

[18] The yield velocity can be estimated using Eq. (1) in Ref. [19]; here we assume a Young modulus of $71 \mathrm{GPa}$ and a Poisson ratio of 0.22 for soda lime glass (from Ref. [20], Table I). For the yield strength we take the value $0.89 \mathrm{GPa}$. This value is the minimum observed strength in soda lime from ASM Engineered Materials Reference Book (Ref. [21]).

[19] J. Schäfer, S. Dippel, and D. E. Wolf, J. Phys. I 6, 5 (1996).

[20] S. F. Foerster, M. Y. Louge, H. Chang, and K. Allia, Phys. Fluids 6, 1108 (1994).

[21] M. L. Bauccio, ASM Engineered Materials Reference Book (ASM International, Materials Park, OH, 1994), p. 324.

[22] J.-M. Hertzch, F. Spahn, and N. V. Brilliantov, J. Phys. II 5, 1725 (1995).

[23] J. A. C. Galles, H. J. Herrmann, T. Pöschel, and S. Sokolowski, J. Stat. Phys. 82, 443 (1996).

[24] J. M. Haile, Molecular Dynamics Simulation (Wiley, New York, 1992).

[25] A. Gervois and D. Bideau, in Disorder and Granular Media, edited by D. Bideau and A. Hansen (Elsevier, Amsterdam, 1993), Chap. 1, pp. 6-9. 